Tome 40, no 25. - Juillet 1933.

\title{
Fütterungsversuche
}

\section{mit unsern einheimischen Ameisenarten}

von

\section{R. ST $\ddot{A G E R}$}

Bern.

Ueber Ernährungsfragen bei den Ameisen im allgemeinen ist man noch recht wenig unterrichtet. Die mediterranen Körnerameisen (Messor, Pheidole, etc.) leben von den eingetragenen Samen, deren Stärke sie vermittelst eines Enzyms in Zucker umwandeln; die mitteleuropäischen Ameisen (Formica, Lasius, Tetramorium, Myrmica, etc.) von tierischer Beute (Insekten aller Art), vom Nektar der Blüten, den süssen Absonderungen der Pflanzenläuse und Lycaenenraupen. Das war bisher so ziemlich alles, was man über dieses Thema wusste. Myrmekologischerseits hatte man sich mit der schönen Sernander'schen Entdeckung ${ }^{1}$, dass nämlich die Ameisen auch den sog. Elaiosomen eifrig nachstellen, fast gar nicht befasst. Und doch ist dieser Nachweis eines Botanikers von grosser Bedeutung, und zwar nicht etwa nur für die Verbreitung der Pflanzen, sondern ebenso sehr für das Leben der Ameisen selbst. Denn die Ameisen bedürfen nebst Eiweiss und Kohlehydraten auch des Fettes, und dieses finden sie eben unter natürlichen Bedingungen in den fettes Oel enthaltenden Anhängseln einer Menge von Samen, bez. Früchten, eben den sog. Elaiosomen. Es liegt nicht in meiner Aufgabe, hier die Sernanderschen Versuche zu wiederholen, obwohl im Laufe dieser Arbeit gegebenenfalls von Fütterung mit elaiosomhaltigen Samen, die ich vorgenommen, gesprochen werden soll. Es handelt sich hier vielmehr darum, an Hand einer Menge von Experimenten im Freien festzustellen, was eigentlich unsere einheimi-

1 Sernander, R. Entwurf einer Monographie der europäischen Myrmekochoren. Kungl. Svenska Vetenskapsakademiens Handlingar, Bol. 41, No 7. Úppsala u. Stockholm, 1906.

Rev. Suisse de Zool., T. 40, 1933. 
sehen Ameisen alles "fressen" und was sie bestimmt ablehnen. Dass sie nicht wahllos alles verzehren, dass sie z. B. gewisse Insekten durchaus bei Seite lassen, habe ich früher schon gezeigt ${ }^{1}$.

Bei der Ausführung von Fütterungsversuchen muss der jeweilige psychologische Zustand der Ameisen beachtet werden; auch die Temperatur hat grossen Einfluss auf das Verhalten der Tiere. Es ist nicht gleichgültig, ob ihnen bei kaltem oder warmem Wetter Nahrung vorgelegt wird. Bei niedriger Temperatur sind z. B. die Formica-Arten träge und kümmern sich wenig um das Eintragen von Beute. Das gleiche ist der Fall bei zu heftiger Sonnenstrahlung. Dann halten sie sich im Schatten auf oder verlassen das Nest überhaupt nicht.

Alle meine Versuche wurden im Freien, an den natürlichen Standorten der betr. Tiere ausgeführt und zwar im Laufe des Sommers 1932 in der Umgebung von Bern, im Val Piora am Ritomsee (Kt. Tessin), im Gebiet des Simplonpasses und auf der Belalp ob Brig (Kt. Wallis).

Von den zu prüfenden Substanzen wurden auf Ameisenstrassen Depots errichtet. Oft auch legte ich die Materialien auf den Nesthaufen, oder wo keine Strassen vorhanden waren, in die Nähe des Nesteinganges und stellte in direkter Beobachtung die betreffenden Vorgänge fest. Meistens wurden zu ein- und demselben Versuch die heterogensten Substanzen verwendet, um aus den Vergleichungen sicherere Schlüsse betreffs der Affinität der Ameisen zu gewissen Stoffen zu ziehen.

A. Fütterungsversuche mit Formica rufa, F. rufo-pratensis, $F$. exsecta und $F$. fusca.

V e r s u c h 1. - In Frieswil (Kt. Bern) am 19. Juni. Gewaltiger Nesthaufen von Formica rufa von $1 \frac{1}{2} \mathrm{~m}$ Durchmesser und ca. $80 \mathrm{~cm}$ Höhe; am Waldrand. Vor einem Gewitter. Sehr schwül. Die Arbeiter tummeln sich zu Tausenden an der Nestoberfläche, gebärden sich aber nicht sehr lebhaft. Ich streue eine Anzahl

${ }^{1}$ Stäger, Rob. Gibt es Insekten, die von Ameisen nicht verzehrt werden? Societas entomologica, 98. Jahrg., Nr. 9. Stuttgart, 1923. - Id. Die Waldameise als Insektenvertilgerin. Zeitschr. f. wiss. Ins. Biol., Bd. XIX, 1924, Heft 3. - Id. Warum werden gewisse Insekten von den Ameisen nicht verzehrt? Zeitschr. f. wiss. Ins. Biol., Bd. XXIV, Nr. 7-8, 1929. 
$\mathrm{R}$ e i s körn e r auf den Haufen. Sofort werden sie überall von den Tieren gepackt und ohne Zögern an die Peripherie des Nestes befördert, wo sie liegen blieben. Keines hatte auch nur den Versuch gemacht, daran zu lecken.

Nun versuche ich es mit zerkleinerten $W$ a $l l n u s s k$ e $\mathrm{n}$ e $n$. Diese werden ganz anders behandelt. Ueberall beginnen die Arbeiter, daran eifrig zu lecken und nach kurzer Zeit verschwinden sie mit ihnen in den Nesteingängen. Dass Ameisen Nüsse lieben, war mir schon von früher her bekannt. Schon im Jahr 1923 sah ich Formicoxenus nitidulus rudelweise an Haselnüssen "fressen » ${ }^{1}$.

Vers u c h 2. - Val Piora (1900 m über Meer), 11. Juli. Formica rufo-pratensis. Nebeliger Tag. Schwaches Leben auf den Haufen. Ich lege $\mathrm{R}$ e is kö $\mathrm{r} n$ e $\mathrm{r}$ auf die Nestoberfläche, die sofort vom Haufen weggetragen werden. W allnuss aber wird sehr lange beleckt und dann in's Nestinnere geschleppt. Zerquetschte Hanfsamen (Cannabis sativa) werden beleckt und ebenfalls eingeheimst.

Ve rs u c h 3. - Val Piora-Fongio, $2212 \mathrm{~m}$, am 11. Juli, mit Formica rufo-pratensis. Wall $\mathrm{nuss}$ und $\mathrm{Arachis}$ werden beleckt und eingetragen; Reis dagegen vom Nest geworfen.

Versuch 4. - Val Piora, am Ritomsee, 1900 m, 17. Juli, mit $F$. rufo-pratensis. Zerkleinerte $\mathrm{P}$ a r a $\mathrm{n} \mathrm{u} \mathrm{s} \mathrm{s}$ wird beleckt und eingetragen.

Versuch 5. - Val Piora, am Ritomsee, 18. Juli. Nebeliger Tag, kalt. Die Arbeiter von F. rufo-pratensis bewegen sich sehr langsam auf Nestoberfläche und Strassen. Ein Depot zerkleinerter Wallnüsse wird auch hier stark beleckt, aber einstweilen bei der Kälte-Lethargie der Tiere nicht eingetragen. Nusstückchen, die ich unmittelbar auf die Oberfläche des Haufens streue, werden allerdings in's Innere befördert.

Vers u c h 6. - Simplon-Kulm, ca. $2000 \mathrm{~m}$, am 19. Juli, mit $F$. rufo-pratensis. Depots auf Strasse gelegt, die sich zwischen Alpenrosen und Heidelbeergebüsch zum Nesthaufen hinzieht. Es

1 StäGer, Rob. Das Leben der Gastameise (Formicoxenus nitidulus Nyl.) in neuer Beleuchtung. Zeitschr. f. Morph. und Oekol. d. Tiere, 3. Band, 2.-3. Heft, 1925. 
werden beleckt und eingetragen: zerquetschter Hanfsamen, Haselnuss, Wallnuss, Mandel, Pinien-Kerne. Ein Depot von Reiskörnern aber wird von der Strasse seitlich abgeführt und unter keinen Umständen in's Nestinnere befördert. Auf diese Strasse hatte ich ferner versuchsweise hingelegt: die Blüten von $\mathrm{H}$ e i d e $\mathrm{l}$ b e e r e n, von denen sofort 15 Stück nach einander in's Nestinnere verschwanden. Eine gleiche Behandlung erfuhren von Seite der Arbeiter Blüten der Silene inflata.

Versuch 7. - Simplon-Kulm, am 20. Juli. Mit F. rufopratensis. Es werden beleckt und eingetragen: Blüten von Heidelbeeren, Preisselbeeren, Lotus corniculatus, Cerastium arvense. Alle diese Blüten werden zuerst mit den Kiefern zerbissen, dann beleckt und endlich in's Nest befördert.

Versuch 8. - Simplon-Kulm, am 21. Juli. Mit F. rufopratensis. Nebeltreiben. Vormittags $10 \mathrm{Uhr}$, bei $10^{\circ} \mathrm{C}$. Temperatur. Von den vorgelegten Blüten von Cerastium arvense, Myosotis alpestris, Thymus serpyllum und Galium pumilum ssp. alpestre, wird nur Cerastium arvense eifrig zerbissen und eingeheimst; die andern werden alle entweder liegen gelassen oder energisch von der Strasse weggeschleppt.

Versuch 9. - Simplon-Kulm, am 22. Juli; Sonne, aber kalter Nordwind. Mit F. rufo-pratensis. Beleckt und auf der Strasse zum und in's Nest getragen werden: Blüten von Polygala chamaebuxus, Trifolium alpinum, Cerastium arvense, Alpenrose und Heidelbeere. Unbeachtet gelassen oder weggeräumt werden: Galium pumilum ssp. alpestre und Myosotis alpestris.

Versuch 10. - Simplon-Kulm, am 22. Juli. Mit F. rufopratensis. Folgende Blüten und Blütenteile werden beleckt und eingetragen: Geranium silvaticum ( $1 / 2$ bis 1 Stunde lang zerbissen, beleckt und dann eingeheimst), Veronica chamaedrys, Cerastium arvense. Von Nest und Strasse fortgeschafft werden: Galium pumilum ssp. alpestre und Nigritella angustifolia.

Es werden ferner eingetragen bez. beleckt: Teigwaren (“Sternli »), mit Olivenöl beschmierte Glasperlen, ebenso mit Zuckersirup armierte Glasperlen.

Ve r s u c h 11. - Simplon-Kulm, am. 22. Juli, mit F. rufopratensis. Zerbissen, beleckt und eingetragen werden: Blüten von 
Cerast. arvense. Ferner beleckt und eingetragen: zuckergetränkte Perlen, olivenölgetränkte Perlen, Teigwaren ("Sternli») und die elaiosomhaltigen Samen von Chelidonium majus (sehr eifrig!).

Versuch 12. - Simplon-Kulm, 23. Juli; grosser rufo-pratensis-Haufen. Von dargebotenen Blüten werden angenommen und eingeheimst: Trifolium badium, Biscutella laevigata, während Androsace chamejasme abgeführt wird. Von andern Stoffen werden lebhaft angenommen: Brotkrume und getrocknete Weinbeeren.

Versuch 13. - Simplon-Kulm, am 24. Juli, mit F. rufopratensis. Blüten von Cerastium arvense werden zerfranst, beleckt und eingetragen. Sehr stark beleckt werden ferner und endlich eingetragen: Eiweiss, Eigelb, Stückchen von Schinken, Gallerich. Reis und Ulmergerste, ob roh oder gekocht, werden dagegen von der Strasse entfernt.

Versuch 14. - Simplon-Kulm, am 26. Juli, mit F. rufopratensis. Es werden eingetragen: Brotkrümchen, Zuckerstückchen, zerquetschte Hanfsamen, Pinienkerne, Wallnuss und Teigwaren. Abgelehnt und weggeführt werden: gekochter Reis und rohe Ulmergerste, die Samen von Crocus vernus (nicht elaiosomhaltig!).

V e rs u ch 15. - Simplon-Kulm, am 26. Juli, mit F. rufopratensis. Teigwaren und Wallnuss werden gierig beleckt und in's Nest abtransportiert.

Versuch 16. - Simplon-Kulm, am 27. Juli, mit F. rufopratensis. Es werden gierig beleckt und eingetragen: Teigwaren und Wallnusskerne. Eine Zeitlang wird beleckt und dann vom Nest weggetragen: Saccharin. Von Anfang an abgelehnt werden: Haferkerne und gekochte und rohe Reis- und Gerstenkörner.

Versuch 17. - Simplon-Kulm, am 28. Juli, mit F. rufopratensis. Saccharin wird immer wieder von neuen Individuen beleckt und immer wieder geflohen. Einzelne tragen kleine Stückchen ein. Im allgemeinen fortgeführt. Schinken, Eiweiss, Eigelb, Gallerich und Brot wird sehr gierig beleckt, lange Zeit und dann restlich noch in's Nestinnere befördert. Gallerich findet gar starken und anhaltenden Zuspruch, sogar grössern als Zuckerstückchen, die doch auch sehr beliebt sind. 
Versuch 18. - Simplon-Kulm, am 28. Juli, mit F. rufopratensis. Beleckt und eingetragen: die Blüten von Trifolium badium, Trif. alpinum, Taraxacum pyrenaicum (Strahlenblüten).

V e r s u ch 19. - Eggen am Simplonpass, ca. $1400 \mathrm{~m}$, am 29. Juli, mit $F$. rufo-pratensis. Intensif beleckt und eingetragen: Speck, Schinken, Brot, Biskuit, Eiweiss, Eigelb, Käse und Zucker. Nicht beleckt und nicht eingetragen: ölgetränkte Glasperlen und Reis.

Versuch 20. - Simplon-Kulm, am 29. Juli, mit F. rufopratensis, grosser Nesthaufen. Die Arbeiter tragen ein: Biskuit, zerquetschte Hanfsamen und Blüten von Cerastium arvense. Sie lassen liegen oder beseitigen: ölgetränkte Glasperlen und unzerquetschte Hanfsamen.

Versuch 21. - Simplon Kulm, am 31. Juli, mit F. rufopratensis, riesiger Haufen von $2 \mathrm{~m}$ Durchmesser, umgeben von einem Kranz ausgeworfener Feinerde und Detritus. Darauf wachsen und blühen: Cerastium arvense, Campanula scheuchzeri, Vaccinien, Juniperus nana und Gramineen. Beleckt und eingetragen: Blüten von Cerastium arvense, Polygala chamaebuxus und Campanula scheuchzeri; ferner Saccharin.

Versuch 22. - Simplon-Kulm, am 31. Juli, mit F. rufopratensis. Beleckt und eingetragen werden: Teigwaren ("Sternli )", Blüten von Cerastium arvense, Strahlenblüten von Chrysanthemum alpinum. Beleckt und teilweise eingetragen: Saccharin.

V e r s u c h 23. - Simplon-Kulm, am 1. August, mit F. rufopratensis. Beleckt und eingetragen werden: Pistazienkerne, Mandel, Wallnuss, Pinienkerne, Blüten von Melampyrum silvaticum, Pinguicula grandiflora- und Geranium silvaticum-Blüten, befeuchtete Zuckerstückchen. Kurz beleckt und zum Teil ein-, zum Teil abgetragen: Saccharin. Beseitigt: Trockene, nicht befeuchtete Stückchen Zucker.

Versuch 24. - Simplon-Kulm, 5. August, mit F. rufopratensis. Temperatur: 9o C. Beleckt und eingetragen: Pinienkerne, Wallnuss, Mandel, zerquetschter Hanfsamen. Beseitigt: Viola biflora-Samen, unzerquetschte Hanfsamen. Gemieden werden die Früchtchen von Anis. 
Versuch 25. - Simplon-Kulm, 10. August, mit F. rufopratensis. Die Arbeiter tragen spontan reichlich die mit einem Elaiosom versehenen Früchte von Thesium alpinum in's Nestinnere. Auf die betr. Strasse gelegt, werden beleckt und eingetragen: Teigwaren, Blüten von Lotus corniculatus. Anhaltend auf der Strasse beleckt, aber nicht eingetragen: Saccharin.

Versuch 26. - Simplon-Kulm, 15. August, mit F. rufopratensis. Beleckt und eingetragen: Wallnuss, Paranuss, Mandel, Pinienkerne. Von der Strasse weggeführt, also beseitigt: die Samen (kein Elaiosom!) von Crocus vernus.

Versuch 27. - Simplon-Kulm, 19. August, mit F. rufopratensis. Stark und lange beleckt und eingetragen: zerquetschte Früchte von Empetrum nigrum.

Versuch 28. - Belalp, $2150 \mathrm{~m}, 22$. August, mit F. rufopratensis. Beleckt und eingetragen: Wallnuss, Mandel, Haselnuss, Pinienkerne. Führen energisch vom Nest und Strasse weg: CrocusSamen.

Versuch 29. - Belalp, 26. August, mit F. rufo-pratensis. Die Arbeiter belecken und tragen ein: Haselnuss, Pistazienkerne, Pinienkerne, Mandel, Paranuss, Arolla-Nüsschen und Pfirsichkerne.

Versuch 30. - Val Piora, am Ritomsee, 12. Juli, mit $F$. exsecta. Es werden beleckt und in's Nest befördert: Wallnuss, zerquetschte Hanfsamen und Samen von Chelidonium majus (mit Elaiosom), die ich von Bern mitgebracht hatte. Von Strasse, bez. Nest beseitigt werden vers chimmelte Samen von Viola odorata (mit Elaiosom).

V e rs u c h 31. - Simplon-Kulm, 31. Juli, mit Formica fusca. Es werden lebhaft beleckt und eingetragen: Wallnuss, Mandel, Teigwaren und zum Teil Saccharin.

Versuch 32. - Simplon-Hohwang, 2200 m, 9. August, mit $F$. fusca. Wir machen an einem kleinen See ein Pic-nic. Spontan holen sich die fusca-Arbeiter die Brosamen, die auf die Erde fallen und tragen sie $8 \mathrm{~m}$ weit in ihr Nest. Vom experimentell vorgelegtem Futter tragen sie ferner ein: Wallnuss, zerquetschten Hanfsamen und Teigwaren. Gemieden wird Fenchel. 
Versuch 33. - Simplon-Kulm, 15. August, mit F. fusca. Es werden beleckt und eingetragen: Paranuss, Pistazienkerne, Pinienkerne, Manna (d. i. die süsse Ausscheidung von Fraxinus ornus). Sofort vom Nest entfernt und weit weggeführt werden die Samen von Crocus vernus.

V e r s u c h 34. - Belalp, 26. August, mit F. fusca. Die Arbeiter belecken und transportieren in's Nest: Paranuss, Haselnuss, Mandel, Pfirsichkerne und Arven-Nüsschen.

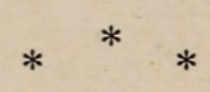

Anhand dieser 34 Versuche erhalten wir folgende Uebersicht:

a) Formica rufa beleckt und trägt ein: Wallnuss. Sie führt vom Nest weg: Reiskörner.

b) Formica rufo-pratensis beleckt und trägt ein: Wallnuss, Haselnuss, Paranuss, Mandel, Pistazien- und Pinienkerne, Arachisfrüchte, Arven-Nüsschen, Pfirsichkerne, zerquetschten Hanfsamen; Brot, Biskuit, Teigwaren (“Sternli )), Eiweiss, Eigelb, Gallerich, Schinken, Speck und Käse; getrocknete Weinbeeren, Früchte von Empetrum nigrum, Zucker (feucht); Blüten von Alpenrose, Heidelbeeren, Preisselbeere, Silene inflata, Lotus corniculatus, Polygala chamaebuxus, Trifolium alpinum, Trifolium badium, Cerastium arvense, Geranium silvaticum, Melampyrum silvaticum, Veronica chamaedrys, Strahlenblüten von Taraxacum pyrenaicum und Chrysanthemum alpinum, Campanula scheuchzeri und Pinguicula grandiflora; Samen von Chelidonium majus und Thesium alpinum; öl- und zuckergetränkte Glasperlen. Sie führt von Nest und Strasse weg: Reis (roh und gekocht), Hafer und Ulmergerste, unzerquetschten Hanfsamen, trockenen Zucker; Blüten von Myosotis alpestris, Androsace chamejasme, Nigritella angustifolia, Galium pumilum ssp. alpestre und Thymus serpyllum; Samen von Viola biflora und Crocus vernus. Sie trägt teilweise, bez. hie und da ein: Saccharin, ölgetränkte Glasperlen, zuckergetränkte Glasperlen.

c) Formica exsecta beleckt und trägt ein: Wallnuss, zerquetschten Hanfsamen, Samen von Chelidonium majus. Sie schafft weg: Verschimmelte Samen von Viola odorata.

d) Formica fusca beleckt und trägt ein: Wallnuss, Mandel, Paranuss, Haselnuss, Arvennüsschen, zerquetschten Hanfsamen, 
Pistazien- und Pinienkerne, Pfirsichkerne, Brot, Manna; teilweise Saccharin. Sie schafft weg: Samen von Crocus vernus. Sie meidet: Fenchelfrüchtchen.

B. Fütterungsversuche mit Myrmica rubida, Myrmica lobicornis, $M$. sulcinodis, Leptothorax acervorum UND Tetramorium caespitum.

Ve r s u c h 35. - Val Piora, 11. Juli, mit Myrmica rubida. Die Arbeiter ergehen sich an der milden Sonne auf der Nestkuppe. Sie belecken und tragen eifrig in's Nestinnere: Wallnuss, Arachis. Auf den Abraum geworfen: Reis.

V e r s u c h 36. - Simplon-Kulm, 28. Juli, mit Myrmica rubida. Sie beleckt und trägt ein: Zuckerstückchen, Schinken, Speck, Wallnuss. Sie lässt unbeachtet oder schafft weg: mit Sesamöl bestrichene Glasperlen, Reis (gekochter) und Gerste.

Ve r su c h 37. - Belalp, 23. August, mit Myrmica rubida. Sie beleckt und trägt ein: Pinien- und Pistazienkerne, Pfirsichkerne. Sie schafft weg vom Nest: Samen von Crocus vernus.

Versuch 38. - Val Piora (Seegarten), 12. Juli, mit Leptothorax acervorum. Es beleckt und trägt ein: Wallnuss, Pistazienund Pinienkerne. Es schafft vom Netz weg: Reis, Samen von Crocus vernus.

Versuch 39. - Simplon-Kulm, 24. Juli, mit Myrmica lobicornis. Sie beleckt und trägt in's Nest: frischen Samen von Viola odorata (Elaiosom !), Paranuss, alle Elemente des käuflichen Vogelfutters (verschiedene Samenarten).

Versuch 40. - Simplon-Kulm, 5. August, mit Myrmica lobicornis. Sie beleckt und trägt ein: Wallnuss, zerquetschten Hanfsamen und Pistazienkerne. Sie meidet streng: Anis-Früchte.

V e r s u c h 41. - Simplon-Kulm, am Rotels-See, 11. August, mit Myrmica sulcinodis. Sie beleckt und trägt ein: Arachis, zerquetschten Hanfsamen und Samen von Viola biflora. Sie lässt unbeachtet liegen: zerquetschten Rübsamen, aus Vogelfutter stammend. 
V e r s u c h 42. - Simplon-Kulm, 12. August, mit Myrmica sulcinodis. Beleckt gierig und trägt ein: Wallnuss, zerquetschten Hanfsamen, Teigwaren ("Sternli ») und Samen von Viola biflora. Nicht eingetragen, bez. vom Nest fortgeschafft wird: Saccharin, Rübsamen. Vor Anis-Körnern schrecken die Ameisen förmlich zurück.

Versuch 43. - Simplon-Kulm, 13. August, mit Myrmica sulcinodis. Sie beleckt und trägt ein: Wallnuss, Mandel, Pistazienund Pinienkerne, Samen von Viola odorata, Teigwaren, ein kleines Stückchen Saccharin. Sie lässt unbeachtet liegen: mit Hanföl getränkte Glasperlen.

V e r s u c h 44. - Belalp, 22. August, mit Myrmica lobicornis. Sie beleckt und trägt ein: Mandel, Pistazienkerne, Haselnuss, Samen von Viola odorata, 3 Samen bez. Früchtchen von Polygonum alpinum. Der Rest dieses Depots aus Polygonum-Samen wird liegen gelassen.

V e r s u c h 45. - Simplon-Kulm, 3. August, mit Tetramorium caespitum. Es beleckt und trägt ein: Wallnuss, Mandel, Arachis, zerquetschte Hanfsamen (leidenschaftlich erfasst). Es schafft weg vom Nest: mit Olivenöl getränkte Glasperlen, Gerste. Es meidet streng: Kümmelfrüchtchen.

V e r s u c h 46. - Simplon-Kulm, 12. August, mit Tetramorium caespitum. Es beleckt und trägt ein: Wallnuss, Arachis, Samen von Viola biflora. Es beleckt mit Hanfsamenöl getränkte Glasperlen, aber trägt sie nicht ein.

V e r s u c h 47. - Belalp, 23. August, mit Tetramorium caespitum. Es beleckt und trägt ein: Paranuss, Wallnuss, Pfirsichkerne.

Fassen wir das Resultat der letzten 13 Versuche zusammen, so ergiebt sich die folgende Uebersicht:

a) Myrmica rubida beleckt und trägt ein: Wallnuss, Arachis, Pinien- und Pistazienkerne, Pfirsichkerne, Zucker, Schinken, Speck. Sie schafft auf die Seite: Reis (roh und gekocht), Gerste, mit Sesamöl getränkte Glasperlen und Samen von Crocus vernus. 
b) Myrmica lobicornis beleckt und trägt ein: Wallnuss, Paranuss, Pistazienkerne, Mandel, Haselnuss, Samen von Viola odorata und V. biflora, alle Elemente des "Vogelfutters", 3 Samen von Polygonum alpinum. Sie lässt unbeachtet liegen: den grösseren Teil des Depots aus Polygonum alpinum-Früchtchen. Sie meidet streng: Anis-Früchtchen.

c) Myrmica sulcinodis beleckt und trägt ein: Wallnuss, Arachis, Mandel, Pistazien- und Pinienkerne, zerquetschten Hanfsamen, Samen von Viola biflora und V. odorata, Teigwaren ("Sternli ») und ein kleines Stückchen Saccharin.

d) Leptothorax acervorum beleckt und trägt ein: Wallnuss, Pistazien- und Pinienkerne. Es schafft vom Nest weg: Reis, Samen von Crocus vernus.

e) Tetramorium caespitum beleckt und trägt ein: Wallnuss, Mandel, Arachis, zerquetschten Hanfsamen, Paranuss, Pfirsichkerne, Samen von Viola biflora, mit Hanfsamenöl getränkte Glasperlen (letztere bloss an Ort und Stelle beleckt). Es schafft bei Seite: mit Olivenöl getränkte Glasperlen. Es meidet streng: Kümmelfrüchtchen.

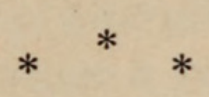

Wenn wir die Zusammenstellungen betrachten, könnten wir leicht $\mathrm{zu}$ falschen Schlüssen gelangen. Es hat den Anschein, dass Formica rufo-pratensis weit mehr Substanzen eintrüge als alle andern aufgeführten Arten zusammen. Das kommt aber einzig daher, dass wir mit $F$. rufo-pratensis sehr viel mehr Versuche ausführten. Hätten wir die Experimente im gleichen Mass mit F. rufa oder $F$. fusca vorgenommen, würden sie an Zahl der eingetragenen Stoffe kaum im Rückstand gewesen sein. Wir wollen nur einen vorläufigen kurzen Ueberblick über die von allen oder den meisten der aufgeführten Arten allgemein beliebten Nahrungsmittel und die des bestimmtesten von ihnen abgelehnten Stoffe geben. Dabei erfahren wir auch bisweilen den Grund des Ablehnens gewisser Materialien.

Auf alle Fälle wird uns klar, dass unsere Ameisen nicht nur von Insekten, Blütennektar und den süssen Absonderungen der Pflanzenläuse und anderer Kerfe leben, sondern dass sie gegebenenfalls 
auf noch ganz andere Dinge erpicht sind. Und da spielen in erster Linie $N$ üsse und $n$ ussartige Früchte eine grosse Rolle. Geradezu gierig machen sich die Arbeiter aller Arten an Wallnüsse. Aber auch Haselnüsse, Paranüsse, Mandeln, Pistazienund Pinienkerne sind nebst Pfirsichkernen und zerquetschten Hanfsamen sehr beliebt und werden eifrig beleckt und in's Nest. eingetragen. Ebenso gesucht sind die elaiosomhaltigen Samen von Chelidonium maius, Viola odorata und $V$. biflora, insofern sie frisch und nicht verdorben (verschimmelt) sind. Alle diese genannten Kerne bez. Samen enthalten f ette $\mathrm{O}$ ele, und diese sind es, was die Ameisen besonders suchen. Was sie in den Elaiosomen im Kleinen finden, das bieten ihnen Nüsse im Grossen. Es ist bisher nicht darauf geachtet worden; aber sicher werden unsere Waldameisen auch spontan Buchnüsschen, aufgebrochene Haselnüsse $u$. dergl. in ihre Bauten schleppen, insofern sie solche an günstigen Stellen finden.

Wie ganz anders wird Reis behandelt! Legen wir z. B. Wallnuss und Reis gleichzeitig vor, wird erstere ebenso stürmisch eingetragen wie letzterer vom Nest entfernt. Da giebt es gar kein Besinnen. Welcher Unterschied zu den Ernteameisen der Mittelmeerländer, die nun wieder ihrerseits gerade Reis leidenschaftlich begehren. Dafür, dass unsere Ameisen mit elaiosomfreien Samen im Allgemeinen nichts anzufangen wissen, gibt der Samen von Crocus vernus ein schönes Beispiel. Welcher Ameisenart wir ihn auch vorlegten, überall wurde er sofort vom Nest entfernt, während Thesium alpinum-Samen, wie wir schon von Sernanders Versuchen her wissen, eben wegen seines Elaiosoms fleissig eingeheimst wird. Anders steht es mit Hanfsamen (Cannabis sativa). Werfen wir sie den Ameisen ganz vor, transportieren sie sie ab. Zerquetschen wir sie aber, werden sie, d. h. deren Inhalt anhaltend beleckt und in's Nestinnere verbracht. Das seltsame Verhalten hängt nun ganz und gar mit dem besondern Bau des Samens zusammen. Er ist nämlich so glatt, dass er den Ameisenkiefern immer wieder entwischt. Sie können ihn nicht aufknacken, so wenig als sie Nüsse aufknacken könnten. Besorgen wir aber für sie diese Arbeit, oder finden sie zertretenen Hanfsamen auf dem Weg, so ist ihnen nichts lieber, als dessen fettreicher Inhalt. Wir müssen also von Fall zu Fall gut zusehen, weshalb ein Versuchsstoff angenommen, ein anderer verworfen wird. 
Sind f e t tes $\mathrm{O}$ e l enthaltende Substanzen für die Ameisen geradezu Leckerbissen, so sind ätherische Oele führende Samen und Früchte ihnen das Verhassteste, was es geben kann. Daher werden Fenchel-, Anis- und Kümmelkörner streng gemieden. Wir kennen den Grund dieser Erscheinung genau; denn wir wissen, dass die ätherischen Oele (wenigstens im geschlossenen Raum) schon in geringsten Geruchsmengen tötlich auf die Ameisen wirken ${ }^{1}$.

Sehr begehrt sind dann ferner eiweisshaltige Stoffe aller Art und in jeder Zubereitung, von Brot, über Biskuit und Teigwaren ("Sternli ») hinweg bis zu Eiereiweiss, Eigelb, Schinken, Käse, etc. Dass unsere Ameisen Zucker lieben, ist längst bekannt. In der freien Natur lassen sie aber bisweilen trockenen Zucker liegen und machen sich erst dahinter, wenn wir ihn anfeuchten. Dass sie aber auch getäuscht werden können, beweist das Lecken und teilweise Eintragen von Saccharin, das doch keine Spur von Nährwert enthält. Der Instinkt ist also auch nicht etwas absolut Sicheres. Es gibt eine Instinkt- Irrung. Weshalb unsere Ameisen so zahlreiche Arten von vorgeworfenen Blüten zerbeissen, belecken und eintragen, ist nicht schwer einzusehen. Es ist der in das Gewebe eingeschlossene $\mathrm{Z} \mathrm{u} \mathrm{c} \mathrm{k} \mathrm{e} \mathrm{r} \mathrm{s} \mathrm{a} \mathrm{f} \mathrm{t,} \mathrm{den} \mathrm{sie} \mathrm{erstreben.} \mathrm{In} \mathrm{hervorra-}$ gender Weise betreibt dieses Geschäft, sozusagen als Spezialistin, eine in der Toscana heimische Ameise (Aphaenogaster testaceopilosa Lucas, spinosa Emery, var. nitida Emery), die im Frühling massenhaft die Blütenblätter der Cistrosen einträgt, um des eingeschlossenen Zuckers habhaft zu werden. Die wieder ausgeworfenen, ausgebeuteten Korollen dieser Pflanzen bilden regelmässig ganze Kränze um das Kraterloch des Nestes herum ${ }^{2}$.

Ausserhalb meiner Versuchsanstellungen sah ich mehrmals, wie Formica rufo-pratensis auf dem Simplon spontan Teile von Blüten auf den Strassen ins Nest transportierten. Eine sehr bevorzugte Blüte ist die von Cerastium arvense, deren zarte, weisse Petalen sie ganz zerreissen und zernagen. Aber auch Polygala chamaebuxus, Silene inflata, Trifolium und andere Blüten werden eifrig ausgebeutet, wohingegen die Blüten von Galium pumilum ssp. alpestre,

${ }^{1}$ StÄGER, Rob. Ueber die Einwirkung von Duftstoffen und Pflanzendüften auf Ameisen. Zeitschr. f. wiss. Ins.-Biologie, Bd. XXVI, Nr. 2-3, 1931.

${ }_{2}$ StÄger, Rob. Resultate meiner Beobachtungen und Versuche an Aphaenogaster testaceo-pilosa Lucas, spinosa Emery, var. nitida Emery. Zeitschr. f. wiss. Ins.-Biologie, Bd. XVIII, 1923. 
Myosotis alpestris und andere mit Vehemenz vor die Tür gesetzt werden. Um den Grund dieses Verhaltens einzusehen, müsste im Einzelnen studiert werden, ob diese nicht akzeptierten Blüten weniger oder keinen Zucker in ihren Geweben enthalten oder ob es vielleicht gewisse Gerüche sind, die die Ameisen abschrecken. Ich vermute, dies letztere möchte bei Galium pumilum ssp. alpestre der Fall sein. Jedenfalls beobachtete ich später einmal, dass auch die Blüten von Achillea Erba-rotta All. ssp. moschata (Wulfen) von den Ameisen verschmäht werden. Die Compositen enthalten bekanntlich oft starke Geruchsstoffe, die auf ätherischen Oelen basieren.

Betreffs Reis, der immer prompt abgeführt wurde, muss hier noch einer besonderen Beobachtung, die ich früher machte, Erwähnung getan werden. Wir wissen durch W. Gozтsch's ${ }^{1}$ und meine eigenen Experimente ${ }^{2}$, dass die Ernteameisen (Messor) der Mittelmeerländer Reis und andere Samen ohne Mälzprozess direkt. verarbeiten und ihre Larven damit füttern, was unsern mitteleuropäischen Ameisen im Allgemeinen, wie wir gesehen, versagt. ist (sie geniessen nur die Elaiosome). Dagegen ist bekannt, dass Tetramorium caespitum und bisweilen Lasius niger in heissen Sommern zu den Allüren der typischen Ernteameisen übergehen können und (auch elaiosomfreie) Samen zur Nahrung einernten und in ihren Nestern aufstapeln. Ich habe dies für Tetramorium caespitum nachgewiesen ${ }^{3}$, das am Obern Aletschgletscher im Sommer 1928 massenhaft die Samen von Trifolium thalii in's Nest. eintrug. Wichtiger war der damalige Nachweis, dass Tetramorium auch wirklich den Inhalt von Samen selbst verzehrt. Dieser Nachweis gelang mir mit Reiskörnern ${ }^{4}$ im künstlichen Nest. Rudelweise wurden sie von den Arbeitern belagert und gierig beleckt, bis sie allerlei Dellen und Deformationen zeigten. Tetra-

${ }^{1}$ Goztsch, W. Beiträge zur Biologie körnersammelnder Ameisen. Zeitschr. f. Morph. und Oekologie d. Tiere, 10. Bd., 2-3, 1928.

${ }^{2}$ StÄger, Rob. Beiträge zur Biologie von Messor barbarus L., Messor instabilis var. bouvieri Bondroit und Pheidole pallidula Nyl. Zeitschr. f. wiss. Ins.-Biologie, Bd. XXIII, 3-4, 1928.

StÄGER, Rob. Weitere Beiträge zur Biologie mediterraner Ameisen. Zeitschr. f. Morph. und Oekologie der Tiere, 15. Bd., 3. Heft, 1929.

${ }^{3}$ StÄGER, Rob. Die Samenverbreitung des Trifolium thalii durch die Rasenameise in der alpinen Stufe. Berichte der Schweiz. Botan. Ges., Heft X X XVIII, 1929.

${ }^{4}$ Loc. cit., p. 211. 
morium caespitum und gelegentlich wohl auch Lasius niger, scheint somit unter unsern einheimischen Ameisenarten eine Sonderstellung einzunehmen und einen biologischen Uebergang zu den typischen Ernteameisen zu bilden, was um so verständlicher ist, als es in den Mittelmeerländern regelmässig Körner einträgt.

Herrn Prof. Rytz am Botan. Instit. Bern verdanke ich bestens die Bestimmung der hier genannten Pflanzen, ebenso Herrn Apotheker Kutter in Flawil die Determination einiger Ameisenarten. 


\section{$2 \mathrm{BHL}$ Biodiversity Heritage Library}

Stäger, R. 1933. "Fütterungsversuche mit unsern einheimischen Ameisenarten." Revue suisse de zoologie 40, 349-363. https://doi.org/10.5962/bhl.part.117660.

View This Item Online: https://www.biodiversitylibrary.org/item/148564

DOI: https://doi.org/10.5962/bhl.part.117660

Permalink: https://www.biodiversitylibrary.org/partpdf/117660

\section{Holding Institution}

American Museum of Natural History Library

\section{Sponsored by}

BHL-SIL-FEDLINK

\section{Copyright \& Reuse}

Copyright Status: In copyright. Digitized with the permission of the rights holder.

Rights Holder: Muséum d'histoire naturelle - Ville de Genève

This document was created from content at the Biodiversity Heritage Library, the world's largest open access digital library for biodiversity literature and archives. Visit BHL at https://www.biodiversitylibrary.org. 